\title{
Attentional prioritizations based on spatial probabilities can be maintained on multiple moving objects
}

\author{
Cary S. Feria \\ San Jose State University, San Jose, California
}

\begin{abstract}
Previous studies have shown that attention prioritizes locations that frequently contain a target. The present study examines whether these spatial prioritizations can be maintained on multiple independently moving objects. Observers viewed two line objects undergoing translational and rotational motion and detected probes appearing on the objects. The probabilities of probes appearing on the centers and ends of objects were manipulated. Experiment 1 showed that attention within moving objects is affected by location probabilities and is also heavily biased toward objects' centers. Experiment 2 showed that if the observer is not informed about location probabilities, the probabilities can be learned. Experiment 3 showed that with multiple-region objects, the center bias is reduced, but the effect of probabilities is unchanged. Experiment 4 showed that two distinct patterns of spatial prioritizations can be maintained on two objects simultaneously. These results suggest that attentional prioritizations based on spatial probabilities can occur in an object-based reference frame.
\end{abstract}

Much research has demonstrated that attending to a spatial location facilitates perceptual processing of a stimulus at that location (e.g., Posner, Snyder, \& Davidson, 1980; Prinzmetal, McCool, \& Park, 2005). For this reason, attention has been compared to a spotlight, enhancing processing of stimuli within an area of the visual field (e.g., Posner et al., 1980). Many studies have shown that the visual system attentionally prioritizes locations on the basis of the likelihood of a target's appearing at those locations. Targets in high-probability locations are detected more efficiently than targets in low-probability locations, which suggests that the visual system strategically allocates its resources to the locations most likely to contain a target (e.g., Geng \& Behrmann, 2002, 2005; Kinchla, 1977; Shaw \& Shaw, 1977).

However, many other studies have suggested that the attentional mechanism selects perceptual objects (e.g., Baylis \& Driver, 1993; Duncan, 1984; Egly, Driver, \& Rafal, 1994; Vecera \& Farah, 1994; for a review, see Scholl, 2001). According to this object-based attentional view, attention is not directed simply to spatial locations in the visual field but to regions that have previously been segmented into objects by the perceptual organization system. A number of studies have shown that space-based and object-based attention can be present concurrently in the same task (e.g., Egly et al., 1994; Shomstein \& Yantis, 2004; Soto \& Blanco, 2004).

Although there is now much evidence that attention can select high-probability locations and that attention can select relevant objects, the interaction between these two mechanisms is less understood. One important question is whether attentional prioritizations based on probabilities can occur in an object-based frame of reference. That is, can attentional prioritizations be encoded relative to positions within individual objects? Relevant to this question, the effects of location probabilities within grouped configurations have been examined in a few studies. Miller (1988) presented observers with a configuration of horizontally aligned letters in which a target letter could occur with high or low probability in each of four locations. After practice, the configuration was shifted in its spatial location to the left or right so that the high- and low-probability locations were at new screen locations. Target detection was facilitated at the high-probability spatial location and at the high-probability relative position within the configuration. This suggests that probabilities facilitate relative positions within configurations, as well as absolute locations in visual space. Hoffmann and Kunde (1999) presented observers with two differently shaped configurations, each with a distinct pattern of target expectancies. They found that the observers adapted different target expectations to the locations in the two configurations. Their results showed stimulus probability effects both for spatial locations and for relative positions within the configuration, although the effect was stronger for the relative positions. Kunde and Hoffmann (2005) found that the impact of the configuration-relative frame of reference increases with increasing spatial uncertainty of targets in absolute spatial coordinates. These studies suggest that attentional prioritizations by probability can

C.S. Feria, cary.feria@sjsu.edu 
occur in a configuration-based, or perhaps object-based, reference frame.

Another critical test of whether these spatial prioritizations are object based is whether the prioritizations can move along with a continuously moving object over time. The purpose of the experiments reported here is to investigate this issue. If the frame of reference for attentional prioritization is object based, then if the object on which the prioritizations occur is moving, the prioritizations should move along with the object. Kahneman and Treisman (1984) proposed that the visual system maintains a continuous representation of each object, even as the object moves and changes. Several studies have supported the idea that attention can follow a moving object. Attentional activation accompanies an object as it moves through space (e.g., Kahneman, Treisman, \& Gibbs, 1992; Lamy \& Tsal, 2000; Soto \& Blanco, 2004). Similarly, inhibition associated with a distracting object follows the object as it moves to a new location (e.g., Ogawa, Takeda, \& Yagi, 2002; Tipper, Brehaut, \& Driver, 1990). These studies suggest that attention accesses object-based representations. Note, however, that some of these studies have shown that after a location on a moving object is cued, attention is enhanced both at the spatial location of the cue and at the new location of the object (Lamy \& Tsal, 2000; Soto \& Blanco, 2004). This suggests that attention may access both an object-based representation and a spatial-coordinate representation simultaneously. Attention can also be maintained on multiple moving objects over time, as has been demonstrated in the multiple-object tracking (MOT) paradigm (e.g., Pylyshyn \& Storm, 1988). In this paradigm, several identical objects move about while the observer attentionally tracks a prespecified subset of the objects, and observers have typically been found to accurately track four or five objects (e.g., Pylyshyn \& Storm, 1988).

There is now ample evidence that attention can follow an object as it moves. However, less is known about how attention is spatially distributed within an attended moving object. Alvarez and Scholl (2005) were the first to examine the spatial distribution of attention within objects moving over an extended period of time. In their study, Alvarez and Scholl used a version of the MOT paradigm in which observers tracked moving lines and small probe dots appeared intermittently at different locations on the lines during the motion. The observers were asked to detect these probes, and the accuracy of the detection of the probes at the different locations was used as a measure of the distribution of attention across the lines. Their results indicated that attention was concentrated at the centers of the lines during tracking.

Feria (2008) used a paradigm similar to that of Alvarez and Scholl (2005) to examine whether spatial probabilities play a role in the distribution of attention within continuously moving objects. In Feria's Experiment 1, during one block of trials, probes were more likely to occur on the centers of the lines, and during another block of trials, probes were more likely to occur on a particular end of each line. Each line was composed of two colored regions to facilitate observers' ability to distinguish which end had the high probability of probe appearances. Overall, probe detection was better at the centers than at the ends, but detection was also affected by probe location probabilities. These results support Alvarez and Scholl's finding that attention is biased toward the centers of moving objects, but also suggest that the distribution of attention within moving objects can be prioritized by spatial probabilities.

Although Feria's (2008) findings suggest that attentional prioritizations based on spatial probabilities can move along with a continuously moving object, they do not definitively establish that the prioritizations occur within an object-based reference frame. Because of the fact that the high-probability end was denoted by a different color, the observers may have been prioritizing attention to a particular color, rather than to a particular spatial location on the object. Much research has shown that attention can select features, such as color (Treisman \& Gelade, 1980; Wolfe, Cave, \& Franzel, 1989). The present study aims to show that attentional prioritizations based on probabilities can occur within a moving object-based reference frame, using uniform objects in which the high-probability location cannot be distinguished by any feature.

If attentional prioritizations based on probabilities can occur within a moving object-based reference frame, another question of interest is whether observers can learn the probability distribution if they are not explicitly informed of it. In many of the previous studies on the effects of spatial probabilities using static displays, observers were not informed of the probabilities, and yet they were able to learn the probability distribution and to prioritize attention accordingly (e.g., Geng \& Behrmann, 2002, 2005; Hoffmann \& Kunde, 1999; Kunde \& Hoffmann, 2005; Miller, 1988). However, some research, in which different tasks were used, has shown that learning of an array at a particular orientation does not transfer to rotated versions of that array (e.g., Chua \& Chun, 2003; Lassaline \& Logan, 1993). This suggests that it may not be possible to learn a distribution of probabilities within a rotating reference frame (i.e., a distribution of probabilities that occur at locations relative to an array or object that is rotating).

Another significant issue is how the distribution of attention within a moving object is affected by the object's perceptual organization. Palmer and Rock (1994; see also Palmer, 1992) proposed that segmentation of a visual scene into objects occurs primarily through the principle of uniform connectedness. According to this principle, a region of homogeneous visual properties (such as color or texture) is organized as a single perceptual unit. A few researchers have investigated whether objects composed of a single region are the basic units that attention selects. In Watson and Kramer's (1999) Experiment 1, they found a strong object-based attention effect with single-region objects, but no object-based effect with multiple-region objects. Matsukura and Vecera (2006) found that when a multiple-region object contains an additional grouping cue, such as an outline surrounding the entire object, an object-based attention effect does occur, although to a lesser extent than with a single-region object. The results of these two studies suggest that multiple-region objects 
are treated by attention as less cohesive units than singleregion objects are. Are the processes of attentional prioritization by probabilities and the attentional center bias affected by whether an object is composed of a single uniform region or of multiple regions? Because prioritization by probabilities and the center bias occur within an objectbased reference frame, when that reference frame is a less perceptually cohesive unit, the effect of probabilities and the center bias are expected to be reduced.

A final topic of interest is whether two different patterns of attentional prioritization can be maintained on two moving objects simultaneously. Using stationary stimuli, Hoffmann and Kunde (1999) and Kunde and Hoffmann (2005) found that observers concurrently established different attentional prioritizations for each of two different configurations. If different patterns of attentional prioritization can be maintained on different moving objects, this would further support the idea that these prioritizations occur within object-based frames of reference.

The objective of the present study is to examine whether attention can maintain spatial prioritizations in multiple independently moving reference frames. In the experiments, observers detected brief probes that appeared on two moving lines. In one condition, the probes were much more likely to occur at the center of each line than at either end. In the other condition, probes were much more likely to occur at one end of each line than at either the center or the opposite end.

Experiment 1 investigated whether attentional prioritizations based on spatial probabilities can occur within a moving object-based reference frame. Experiment 2 tested whether spatial probability distributions within moving objects can be learned if the observer is not given information about the probabilities. Experiment 3 examined whether the attentional distribution within a moving object is affected by the cohesiveness of the object. Experiment 4 assessed whether attention can maintain two different patterns of spatial prioritizations on two moving objects simultaneously.

\section{EXPERIMENT 1}

The purpose of Experiment 1 was to investigate whether attention can maintain spatial prioritizations on moving objects over time. If the reference frame for attentional prioritization is object based, then when the object on which the prioritizations occur is moving, the prioritizations should follow the object as it moves. Feria (2008) found that attention was directed to high-probability locations on moving objects, but in that experiment, the high-probability locations were designated by color, so attention may have been allocated to a particular color, rather than to a particular spatial location on an object. Thus, Experiment 1 aimed to test whether attention is directed to high-probability locations on moving objects when these locations do not correspond with any particular object feature.

The observers here viewed two lines undergoing translational and rotational motion (see Figure 1). Intermittently during the motion, gray circular probes appeared briefly at either the center of a line or near one of its end- points. The observers detected these probes by pressing a key on the keyboard.

In one block of trials, the probes were more likely to appear at each line's center with a probability of occurrence of .8 at the center and 11 at each of the ends. In the other block of trials, the probes were more likely to appear at a particular end of each line, with a probability of . 8 at that end, .1 at the opposite end, and . 1 at the center. The observers were informed of these probabilities, and at the beginning of each trial, before the lines began to move, one third of the line was drawn briefly in green, to indicate the high-probability location on that trial. This was helpful primarily for the end high-probability condition, so that the observers were aware of which end of each line would have the high probability of probe appearances on that trial. However, in order for there to be no possibility of attention's being directed to features that are correlated with probe probabilities, the green color disappeared before the lines began to move and probes began appearing. Thus, during the $90 \mathrm{sec}$ in which the lines were moving and the probes were appearing, the lines were drawn in uniform white color.

If spatial prioritizations can occur within a moving object-based reference frame, observers' probe detection should be improved at high-probability locations on the objects. Alternatively, if spatial prioritizations cannot occur within an object-based reference frame, and Feria's (2008) results were simply due to observers attending to particular colors, then the observers' probe detection should not be affected by the probability manipulation.

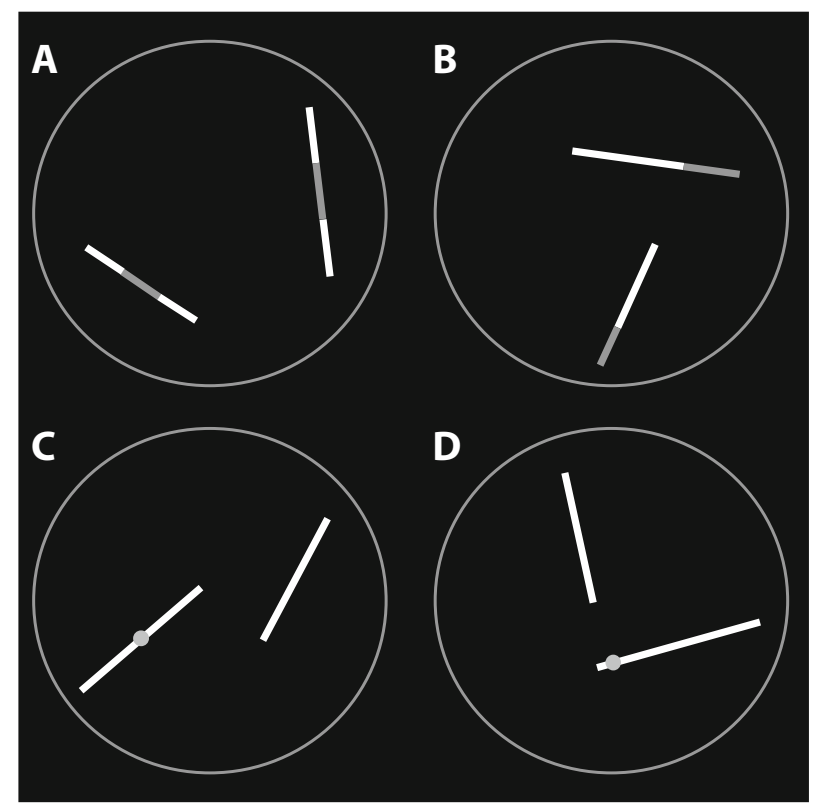

Figure 1. Examples of stimuli used in Experiment 1 (not drawn to scale). During the stationary phase, either the center of each line (A) or an end of each line (B) was highlighted to indicate the high-probability location. During the motion phase, the highlighting disappeared, and probes appeared on the centers of the lines $(C)$ and the ends of the lines (D). The green highlighting in the actual displays is shown here as gray. 


\section{Method}

Observers. The observers were 20 undergraduate students from San Jose State University. Each participated in a 1-h session and was compensated with course credit. All of the observers had selfreported normal or corrected-to-normal vision. None of the observers was familiar with the purposes of the experiment.

Apparatus. The observers were seated in a darkened room approximately $75 \mathrm{~cm}$ from the display. The stimuli were presented on a 20-in. (50-cm) flat-screen CRT monitor with a resolution of 1,920 $\times$ 1,440 pixels, controlled by a Dell Precision workstation. The experimental procedure was generated in $\mathrm{C}++$, using the OpenGL libraries.

Stimuli. On each trial, two lines were presented on a black background (see Figure 1). Each line had a width of $0.02^{\circ}$ visual angle ( 1 pixel). At the beginning of each trial, two lines were presented, each of which was randomly assigned a length between $1.88^{\circ}$ and $5.89^{\circ}$, a starting location, and a starting orientation. One endpoint of each line was arbitrarily designated as End 1 and the other endpoint as End 2. For the first $2.5 \mathrm{sec}$ of the trial, the lines were stationary. During this stationary phase, one third of each line was drawn in green, and the other two thirds of each line were drawn in white. The green area indicated the location on each line that would have the high probability of probe appearances on that trial. Thus, the green one-third area of each line could be on either the center of the line or one of the ends. Then the green color disappeared, leaving the entire line drawn in white, and the motion phase began. The motion phase lasted for $90 \mathrm{sec}$, during which each line translated and rotated. Each line had a translation speed of $0.40 \% \mathrm{sec}$. The $x$ - and $y$-components of the translation speed were chosen randomly for each line, with the constraint that both the $x$ - and the $y$-components had to be at least $0.08^{\circ} / \mathrm{sec}$. In addition, each line had a rotation speed of $0.04^{\%} / \mathrm{sec}$ (degrees here refers to angle of rotation). Each of these speed components remained constant throughout the trial. Lines could intersect each other. The display area was bounded by a gray outline circle with a diameter of $14.6^{\circ}$. The line composing the circle was $0.03^{\circ}$ in width ( 2 pixels). There was a buffer with a width of $0.16^{\circ}$ (10 pixels) on the inside of the circle, so that the line segments would not touch the circle. Whenever a line reached the edge of the display, its motion was reversed to send it back into the display area.

During the motion phase, several gray probe disks were presented on the lines. Each probe had a diameter of $0.13^{\circ}$ and was presented for $215 \mathrm{msec}$. Only one probe was presented at a time, and a random interval of 1,000-2,200 msec passed between the onset of a probe and the onset of the next probe. The first probe appeared between 1,000 and 2,200 msec after the motion began. The last probe appeared at least $1,000 \mathrm{msec}$ before the end of the motion. The locations in which the probes appeared were at the center of a line and near either of the endpoints of a line. Endpoint probes were inset $0.13^{\circ}$ (one probe diameter) toward the center of the line

The probe probability conditions were presented within subjects, in separate blocks. In one block (the center high-probability condition), the probes appeared on the center with a probability of .8 , on End 1 with a probability of .1, and on End 2 with a probability of .1 . In the other block (the End 1 high-probability condition), the probes appeared on the center with a probability of .1, on End 1 with a probability of .8, and on End 2 with a probability of .1. The order of these conditions was counterbalanced.

Procedure. The observers were instructed to press a key as quickly as possible upon seeing a probe. Only keypresses within $1,000 \mathrm{msec}$ of a probe onset were counted as hits. All other keypresses were considered false alarms. The observers were informed of the probabilities of the probes' occurring in each of the locations on the lines and that the green area would indicate the highprobability locations on each trial.

At the end of the motion phase, the observers were given feedback about the percentage of the probes that they had detected on that trial. If 10 or more false alarms had been made on a given trial, the observer was penalized by having to wait $30 \mathrm{sec}$ at this point, and the trial was considered invalid. After viewing the feedback, the observers pressed a key to move on to the next trial.

In summary, each trial consisted of the 2.5 -sec stationary phase during which the lines were shown with highlighting, the 90-sec motion phase during which the lines moved and the probes appeared, and the feedback.

Design. The independent variables were probe location (center, End 1, End 2) and high-probability location (center, End 1). Both of these variables were run within subjects. The dependent variable was the probe detection miss rate. This was defined as the number of probes that were not detected divided by the number of probes presented. In the data analysis, each probe event was considered independently and without regard to the trial from which it came. Each observer completed two blocks. Each block consisted of four practice trials and eight experimental trials. Within each block, the trials were presented in a random order for each observer.

\section{Results}

An average of 55.5 probes were presented per experimental trial, resulting in an average of 889 probes per observer. The smallest number of probes that occurred on any trial for any observer was 51 , and the greatest number was 62 . Only trials on which no more than 9 false alarms were recorded were included in the probe detection analyses. Thus, an average of 0.6 trials, or 33.2 probe events, were excluded per observer. An average of 1.9 false alarms occurred per tracking trial for trials included in the probe detection analyses. The mean detection miss rate was calculated for each observer for each of the six conditions and was submitted to a 3 (probe location) $\times$ 2 (high-probability location) repeated measures ANOVA.

The main effect of probe location was significant $[F(1.8,34.3)=98.55, p<.001]$. (Note that all tests of within-subjects independent variables with more than two levels were adjusted using the Greenhouse-Geisser procedure to compensate for possible deviations from sphericity.) Bonferroni post hoc tests were used to test for differences between the locations. Miss rates were lower for probes occurring on the center $(M=.22, S D=.09)$ than for those on End $1(M=.38, S D=.11)(p<.001)$. Miss rates were also lower for probes occurring on the center than for those on End $2(M=.44, S D=.13)(p<.001)$. Miss rates were also lower for probes on End 1 than for those on End $2(p<.01)$.

The main effect of high-probability location was not significant $[F(1,19)=1.98, p>.05]$. The miss rates in the End 1 high-probability condition $(M=.34, S D=.09)$ did not differ from those in the center high-probability condition $(M=.36, S D=.13)$.

Importantly, a significant interaction was found between probe location and high-probability location $[F(1.8,34.8)=4.95, p<.05]$. This interaction can be seen in Figure 2. Bonferroni comparisons indicated that in the center high-probability condition, the center probes had lower miss rates than either the End 1 probes $(p<$ $.001)$ or the End 2 probes $(p<.001)$, whereas the End 1 probes did not differ from the End 2 probes $(p>.05)$. However, in the End 1 high-probability condition, the End 1 probes had lower miss rates than the End 2 probes $(p<.001)$ but higher miss rates than the center probes $(p<.001){ }^{1}$ 


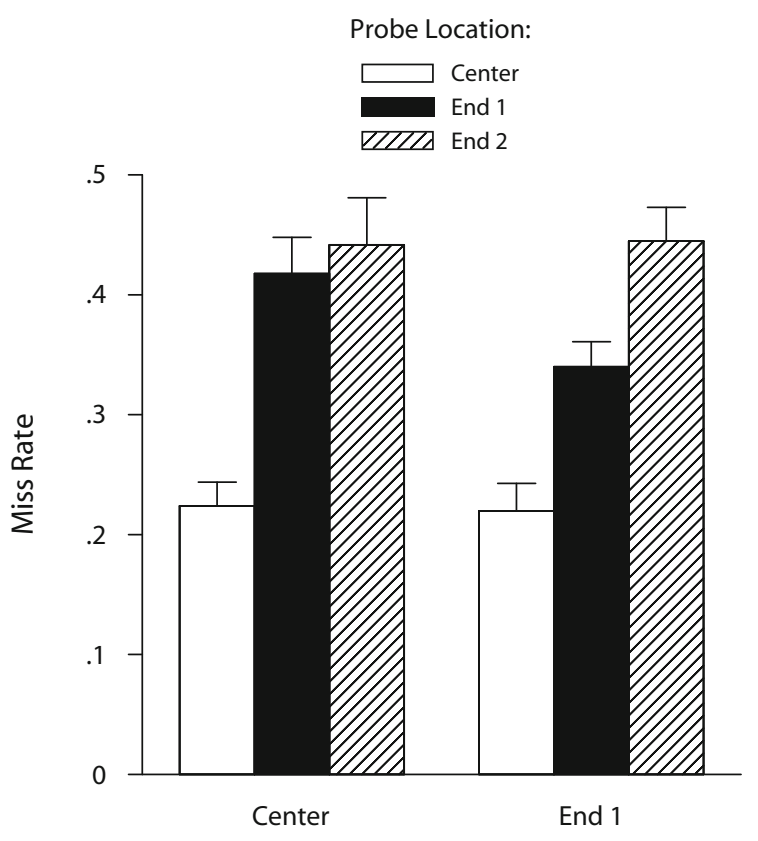

High-Probability Location

Figure 2. Miss rate as a function of high-probability location and probe location in Experiment 1. Error bars represent the standard error.

\section{Discussion}

In Experiment 1, probe detection performance was affected by probe probabilities and was also enhanced at the objects' centers. The finding that probe detection was facilitated at high-probability locations suggests that attention can maintain spatial prioritizations on moving objects over time. This occurred even though the highprobability locations were not denoted by any particular object feature, so, unlike in Feria (2008), the effect cannot be explained by attention to particular object features. Detection of probes on the center was not improved when the center was the high-probability location (Bonferroniadjusted $p>.05$ ); however, this finding was not consistent across the four experiments.

The attentional advantage for the centers of objects was sizable. Probe detection was better on object centers than on the ends when the center was the high-probability location and, by a reduced margin, when an end was the high-probability location. This is consistent with Alvarez and Scholl's (2005) and Feria's (2008) finding of a bias toward the centers of moving objects. Moreover, Feria found that the center bias occurs with stationary objects and that translation, rotation, and size-change motions do not affect the magnitude of the center bias. Other researchers using stationary arrays and objects also previously found that attention (Zhou, Chu, Li, \& Zhan, 2006) and eye movements (Vishwanath \& Kowler, 2003, 2004) are biased toward the center of an array or object.

The results of this experiment showed that the attentional prioritization by probabilities followed each object as it moved, which indicates that these prioritizations can occur within a moving object-based reference frame.

\section{EXPERIMENT 2}

Experiment 1 demonstrated that spatial prioritizations can follow an object as it moves if the observer is informed of the probability distribution. But what if the observer has no knowledge of the probability distribution? Can observers learn the distribution of location probabilities on a moving object and prioritize attention accordingly? The purpose of Experiment 2 was to determine whether attentional prioritizations can be learned within a moving object-based reference frame if the observer is not given any information about the location probabilities. In many previous studies on the effect of location probabilities in which stationary displays were used, observers were given no information about the probabilities, and yet they were able to learn the probabilities and to prioritize attention to high-probability locations (e.g., Geng \& Behrmann, 2002, 2005; Hoffmann \& Kunde, 1999; Kunde \& Hoffmann, 2005; Miller, 1988). On the other hand, some researchers have found that when learning of an array occurs at a particular orientation, that learning does not transfer to different orientations of the array (Chua \& Chun, 2003; Lassaline \& Logan, 1993). Thus, learning of probability distributions may not be possible within rotating and translating reference frames.

In Experiment 2, observers were not given any information about the location probabilities. In one block of trials, the probes were more likely to appear at each line's center, with a probability of occurrence of .9 at the center and .05 at each of the ends. In the other block of trials, the probes were more likely to appear at a particular end of each line, with a probability of .9 at that end, .05 at the opposite end, and .05 at the center. Unlike in Experiment 1 , observers were not told these probabilities, and there was no green color at the beginning of the trial to indicate high-probability locations. The lines were always uniformly white.

If observers can learn a distribution of location probabilities on a rotating and translating object and prioritize their attention according to the probabilities, the observers' probe detection should be improved at high-probability locations on the objects, as in Experiment 1. However, if it is not possible to learn location probability patterns on an object when the object is rotating and translating, the observers' probe detection should not be affected by the probability manipulations.

\section{Method}

Observers. The observers were 18 undergraduate students from San Jose State University. Each participated in a 1-h session and was compensated with course credit. All of the observers had selfreported normal or corrected-to-normal vision. None of the observers was familiar with the purposes of the experiment.

Apparatus. The apparatus was the same as that in Experiment 1.

Stimuli. The stimuli were the same as those in Experiment 1, with the following exceptions. The stationary phase lasted for $2 \mathrm{sec}$, and during this time, the lines were drawn entirely in white. There was no 
green coloring to indicate the high-probability location. The lengths of the lines were randomly assigned between $1.57^{\circ}$ and $9.42^{\circ}$. During the motion phase, each line had a translation speed of $0.26^{\circ} / \mathrm{sec}$. The $x$ - and $y$-components of the translation speed were chosen randomly for each line, with the constraint that both the $x$ - and the $y$-components had to be at least $0.05^{\circ} / \mathrm{sec}$. Rotational motion was the same as in Experiment 1. The display area was bounded by a gray-outlined rectangle, subtending $13^{\circ} \times 10^{\circ}$. The line composing the rectangle was $0.03^{\circ}$ in width (2 pixels). There was a buffer with a width of $0.16^{\circ}(10$ pixels $)$ around each line of the rectangle, so that the line segments would not touch the rectangle.

Probe probability conditions were presented within subjects, in separate blocks. In one block (the center high-probability condition), the probes appeared on the center with a probability of .9 , on End 1 with a probability of .05 , and on End 2 with a probability of .05 . In the other block (the End 1 high-probability condition), the probes appeared on the center with a probability of .05, on End 1 with a probability of .9 , and on End 2 with a probability of .05. The order of these conditions was counterbalanced.

Procedure. The procedure was the same as that in Experiment 1, except that the observers were given no information about the probabilities of the probes' occurring in each of the locations on the lines.

Design. The design was the same as that in Experiment 1.

\section{Results}

An average of 55.6 probes were presented per experimental trial, resulting in an average of 890 probes per observer. The smallest number of probes that occurred on any trial for any observer was 51, and the greatest number was 60. Again, only trials on which no more than 9 false alarms were recorded were included in the probe detection analyses. Thus, an average of 0.7 trials, or 36.7 probe events, were excluded per observer. An average of 2.1 false alarms occurred per tracking trial for trials included in the probe detection analyses.

An ANOVA showed a significant main effect of probe location $[F(1.7,29.0)=50.83, p<.001]$. Bonferroni post hoc tests were used to test for differences between the locations. Miss rates were lower for the probes occurring on the center $(M=.14, S D=.06)$ than for those on End $1(M=.35, S D=.11)(p<.001)$. Miss rates were also lower for the probes occurring on the center than for those on End $2(M=.41, S D=.15)(p<.001)$. The miss rates for the probes at End 1 and End 2 did not differ $(p>$ $.05)$.

The main effect of high-probability location was not significant $[F(1,17)=2.01, p>.05]$. The miss rates in the End 1 high-probability condition $(M=.28, S D=.09)$ did not differ from those in the center high-probability condition $(M=.32, S D=.12)$.

Notably, as in Experiment 1, a significant interaction was found between probe location and high-probability location $[F(1.8,31.2)=20.65, p<.001]$. This interaction can be seen in Figure 3. Bonferroni comparisons indicated that in the center high-probability condition, center probes had lower miss rates than either End 1 probes $(p<.001)$ or End 2 probes $(p<.001)$, whereas End 1 probes did not differ from End 2 probes $(p>.05)$. However, in the End 1 high-probability condition, End 1 probes had lower miss rates than End 2 probes $(p<.001)$ but higher miss rates than center probes $(p<.01)$.

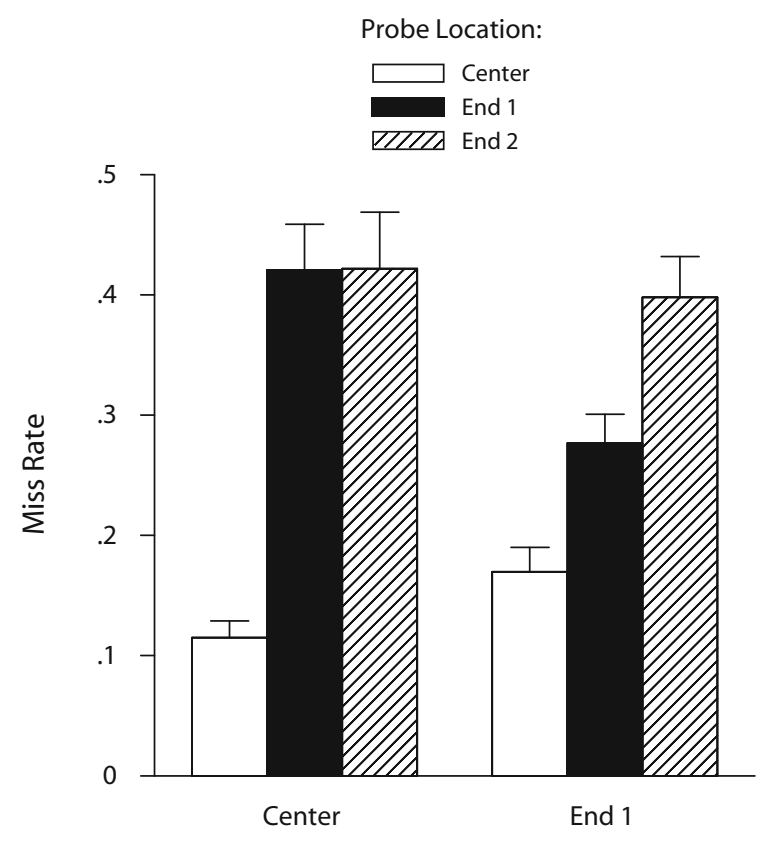

High-Probability Location

Figure 3. Miss rate as a function of high-probability location and probe location in Experiment 2.

\section{Discussion}

The results of Experiment 2 were similar to those of Experiment 1, even though in Experiment 2 the observers were not given any information about the probe probability distribution. The finding that probe detection was still facilitated at high-probability locations demonstrates that observers can learn a distribution of location probabilities on rotating and translating objects and can prioritize attention according to the probabilities. Spatial probabilities can be learned within a moving object-based reference frame.

This result indicates that learning of probability distributions is not limited to cases in which the orientation angle of the reference frame remains constant. In this experiment, the objects were constantly moving, making it unlikely that a probe would occur when the object had the same orientation and location as previously. Prior studies have shown that when an array is only learned at a particular orientation angle, the learning does not transfer to different rotated orientations of the array (Chua \& Chun, 2003; Lassaline \& Logan, 1993). However, Experiment 2 shows that learning of a spatial probability distribution can occur even when the reference frame varies in orientation and location across probe appearances.

In order to assess whether the learning of the probability distributions was implicit or explicit, the observers were asked during the debriefing after the experiment whether they believed that the probes had occurred more on particular locations on the lines during each of the two blocks. All but 1 of the observers reported the probability pattern correctly (i.e., center or end high probability) for at least 
one of the two blocks. However, 3 of the observers were either unaware of or incorrectly identified the distribution in one of the two blocks. The high level of awareness among most of the observers suggests that the learning of the probability distributions was typically explicit.

The attentional bias toward the centers of objects was evident in Experiment 2 as well. Unlike in Experiment 1, however, probe detection on centers improved when the center was the high-probability location (Bonferroniadjusted $p<.05$ ).

\section{EXPERIMENT 3}

Experiments 1 and 2 found that the distribution of attention within a moving object is affected by two factors: prioritization of high-probability locations and a center bias. The purpose of Experiment 3 was to examine whether these two factors are affected by the cohesiveness of the object.

Palmer and Rock (1994) proposed that single uniform regions are the basic objects of perceptual organization. Other studies have shown that objects composed of multiple regions either are not treated by attention as cohesive units (Watson \& Kramer, 1999) or are treated as less cohesive units than single-region objects are (Matsukura $\&$ Vecera, 2006). Does uniform connectedness affect the center bias? Alvarez and Scholl (2005) proposed that the attentional bias toward the centers of objects may be due to the need to represent the location of an extended object at a single point. The process of determining this summary location, and biasing attention toward it, seems likely to require the use of the object representation produced by the perceptual organization principles. Thus, a multipleregion object, which has a less cohesive object representation than a single-region object does, is expected to produce a weaker bias toward its center point than does a single-region object.

Does uniform connectedness affect attentional prioritization by probabilities? Experiments 1 and 2 showed that spatial prioritizations occur within an object-based reference frame. One hypothesis is that if the object-based reference frame has a less cohesive object representation, there will be less ability to maintain attentional prioritizations within that reference frame. Thus, a multiple-region object would be expected to produce a weaker effect of spatial prioritizations than a single-region object.

On the other hand, the top-down demands of attentional prioritization by probabilities may override the less cohesive perceptual organization of multiple-region objects. Watson and Kramer's (1999) Experiment 2 demonstrated that multiple-region objects produce an object-based effect when the task requires attention to the object as a whole. This suggests that top-down task demands play a role in whether the unit that attention selects consists of a single region or multiple regions. In the task of the present experiments, the observers needed to use an object-based reference frame in order to optimally prioritize attention to detect the probes. Thus, the top-down task demands encourage selection of the object as a whole, and this may supersede the fact that the object consists of multiple regions. According to this hypothesis, the process of spatial prioritization in an object-based reference frame will select a multiple-region object in its entirety. Thus, a multipleregion object will produce the same magnitude of spatial prioritization effect as a single-region object does.

Experiment 3 was identical to Experiment 1, except that each line was composed of several segments, alternating in color (see Figure 4). If prioritization by probabilities and the center bias are not affected by the cohesiveness of the object representation, then in Experiment 3 the observers' probe detection will be facilitated at high-probability locations and on objects' centers to the same extent as in Experiment 1 . However, if prioritization by probabilities and/or the center bias are affected by the cohesiveness of the object representation, facilitation at high-probability locations and/or objects' centers will be reduced in Experiment 3.

\section{Method}

Observers. The observers were 18 undergraduate students from San Jose State University. Each participated in a 1-h session and was compensated with course credit. All of the observers had selfreported normal or corrected-to-normal vision. None of the observers was familiar with the purposes of the experiment.

Apparatus. The apparatus was the same as that in Experiment 1.

Stimuli. The stimuli were the same as those in Experiment 1, with the following exceptions. Each line was composed of five equally sized segments, alternating in color. The two segments at the ends of the line and the segment at the middle of the line were drawn in

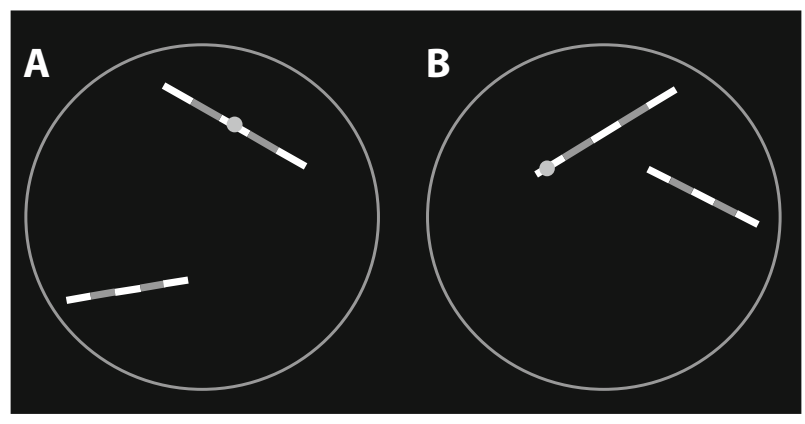

Figure 4. Examples of multiple-region lines presented during the motion phase in Experiment 3 (not drawn to scale). (A) Lines with a center probe. (B) Lines with an end probe. 
white. The other two segments were drawn in a gray color that was darker than the probes. The probes never appeared on the gray segments of the lines.

Procedure and Design. The procedure and design were the same as those in Experiment 1.

\section{Results}

An average of 55.8 probes were presented per experimental trial, resulting in an average of 892 probes per observer. The smallest number of probes that occurred on any trial for any observer was 51 , and the greatest number was 60 . Again, only trials on which no more than 9 false alarms were recorded were included in the probe detection analyses. Thus, an average of 0.2 trials, or 9.0 probe events, were excluded per observer. An average of 1.5 false alarms occurred per tracking trial for trials included in the probe detection analyses.

An ANOVA showed a significant main effect of probe location $[F(1.6,27.9)=18.98, p<.001]$. Bonferroni post hoc tests were used to test for differences between the locations. The miss rates were lower for the probes occurring on the center $(M=.39, S D=.11)$ than for those on End $1(M=.45, S D=.13)(p<.01)$. The miss rates were also lower for the probes occurring on the center than for those on End $2(M=.51, S D=.17)(p<.001)$. The miss rates were also lower for the probes on End 1 than for those on End $2(p<.05)$.

The main effect of high-probability location was not significant $[F(1,17)=0.01, p>.05]$. The miss rates in the End 1 high-probability condition $(M=.45, S D=.14)$ did not differ from those in the center high-probability condition $(M=.45, S D=.15)$.

Critically, a significant interaction was found between probe location and high-probability location $[F(1.3,22.7)=8.22, p<.01]$. This interaction can be seen in Figure 5. Bonferroni comparisons indicated that in the center high-probability condition, the center probes had lower miss rates than either the End 1 probes $(p<.001)$ or the End 2 probes $(p<.001)$, whereas the End 1 probes did not differ from the End 2 probes $(p>.05)$. However, in the End 1 high-probability condition, the End 2 probes had higher miss rates than either the End 1 probes $(p<$ $.05)$ or the center probes $(p<.05)$, whereas the End 1 probes did not differ from the center probes $(p>.05)$. This result differed from those of Experiments 1 and 2, in which, in the End 1 high-probability condition, the End 1 probe miss rates were intermediate between the End 2 and the center miss rates.

Comparisons between Experiment 1 and Experiment 3. A separate analysis was conducted to compare the magnitude of the center bias in Experiments 1 and 3. The magnitude of the center bias was calculated for each observer as the average miss rate on End 1 (combining both blocks) minus the average miss rate on the center (combining both blocks). The center bias was significantly smaller in Experiment $3(M=.07, S D=.06)$ than in Experiment $1(M=.16, S D=.06)[t(36)=4.49, p<$ $.001]$. However, a single-sample $t$ test showed that in Experiment 3 , the center bias was still significantly greater than zero $[t(17)=4.41, p<.001]$.

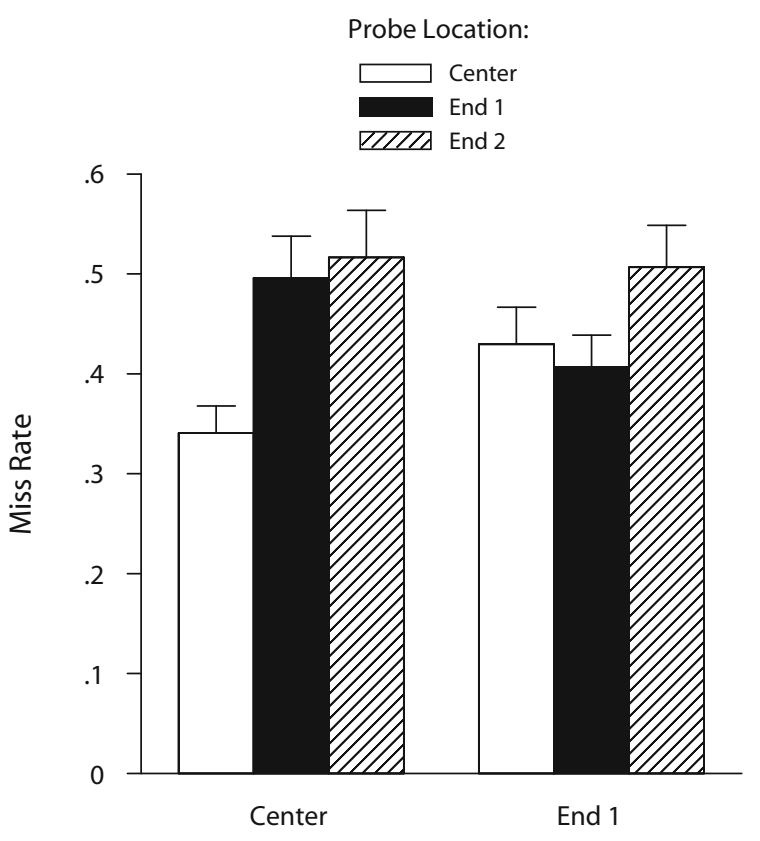

High-Probability Location

Figure 5. Miss rate as a function of high-probability location and probe location in Experiment 3.

An additional ANOVA was conducted to compare the magnitude of the effect of probabilities (measured by the probe location $\times$ high-probability location interaction) in Experiments 1 and 3. The data from Experiments 1 and 3 were subjected to an ANOVA, with probe location and high-probability location as within-subjects factors and experiment as a between-subjects factor. The experiment $X$ probe location $X$ high-probability location interaction was not significant $[F(1.7,62.2)=2.79, p=.08]$. The marginally significant trend was in the direction of a greater effect of probabilities in Experiment 3. There was also a significant main effect of experiment $[F(1,36)=$ $7.14, p<.05]$, indicating a higher overall miss rate in Experiment 3 than in Experiment 1.

\section{Discussion}

The results of Experiment 3 were similar to those of Experiments 1 and 2 , in that probe detection was facilitated at high-probability locations and centers of objects. However, the magnitude of the center bias was smaller in Experiment 3 than in Experiment 1, demonstrating that multipleregion objects produce less center bias than do singleregion objects. This shows that the strength of the center bias is graded, with less perceptually cohesive objects producing less center bias. This result is consistent with the idea that the process of representing the location of the object at a single center point and biasing attention to that point requires the use of the object representation based on the perceptual organization principles. For multiple-region objects, the object representation is less cohesive, and thus, less bias toward the center point occurs. 
On the other hand, the effect of probabilities did not differ significantly between Experiments 1 and 3, demonstrating that attentional prioritization by probabilities is not reduced for multiple-region objects. Experiments 1 and 2 showed that spatial prioritizations occur within an objectbased reference frame. It might be expected that when the reference frame is a less cohesive unit, there would be less ability to maintain spatial prioritizations in that reference frame. However, this did not occur. Rather, the results are consistent with the hypothesis that when observers need to use an object-based reference frame to optimally prioritize attention, the top-down demands cause the object to be selected as a whole, overriding the lack of uniform connectedness. This finding is consistent with previous research showing that when top-down task demands require attention to an entire object, a multiple-region object is selected as a single unit (Watson \& Kramer, 1999).

The miss rate was higher overall in Experiment 3 than in Experiment 1. The finding of a higher miss rate with multiple-region objects than with single-region objects is consistent with Olson and Jiang's (2002) finding that maintaining objects in visual short-term memory (VSTM) requires more resources if each object comprises two colors than if each object comprises a single color.

It could be contended that the gray segments of each line may have been perceived as part of the black background and, thus, that the three white segments of each line were perceived as three separate objects. Consequently, the reduction in the center bias in Experiment 3 could be due to each line's being perceived as a group of three objects, rather than as a single object as in Experiment 1. Future experiments could systematically vary the luminance difference between the gray and white segments of the lines to ascertain how this affects the magnitude of the center bias and to determine whether there is a threshold luminance difference at which the line is no longer perceived as a single object.

\section{EXPERIMENT 4}

Experiments 1-3 have indicated that observers can prioritize attention on the basis of location probabilities on two objects that are moving. However, at any given time, both objects in view had similar location probability patterns: Either both lines had high probability of probes on their centers, or both lines had high probability on one of their ends. Could two entirely different patterns of attentional prioritization be maintained on two moving objects simultaneously? Using static stimuli, Hoffmann and Kunde (1999) and Kunde and Hoffmann (2005) found that observers simultaneously established two different probability distributions in two separate spatial configurations. If different patterns of attentional prioritization can be maintained on different moving objects, this would further support the idea that these prioritizations occur within object-based reference frames.

In Experiment 4, on each trial, each of the two lines had a different probability distribution. On one line, the probes were more likely to appear at its center, with a probability of occurrence of .9 at the center and .05 at each of the ends. On the other line, the probes were more likely to appear at one of its ends, with a probability of .9 at that end, .05 at the opposite end, and .05 at the center. The observers were informed of these probabilities, and at the beginning of each trial, one third of each line was drawn in green briefly, to indicate the high-probability location on each line. The green color disappeared, leaving the lines uniformly white, before the lines began to move and probes began appearing. If different patterns of attentional prioritization can be concurrently maintained on different objects, the observers' probe detection should be improved at the high-probability location on each object, yielding a pattern of data similar to those in Experiments 1 and 2.

\section{Method}

Observers. The observers were 20 undergraduate students from San Jose State University. Each participated in a 1-h session and was compensated with course credit. All of the observers had selfreported normal or corrected-to-normal vision. None of the observers was familiar with the purposes of the experiment.

Apparatus. The apparatus was the same as that in Experiment 1.

Stimuli. The stimuli were the same as those in Experiment 1, with the following exceptions. During the stationary phase, one line had the green-colored one-third area at its center, and the other line had the green-colored one-third area on one of its ends. The stationary phase lasted for $3 \mathrm{sec}$.

On each trial, each of the two lines had a different probability distribution. On one line (the center high-probability line), the probes appeared on the center with a probability of .9 , on End 1 with a probability of .05 , and on End 2 with a probability of .05 . On the other line (the End 1 high-probability line), the probes appeared on the center with a probability of .05 , on End 1 with a probability of .9 , and on End 2 with a probability of .05 .

Procedure. The procedure was the same as that in Experiment 1.

Design. The design was the same as that in Experiment 1, with the following exceptions. Each observer completed two blocks. The first block consisted of four practice trials and nine experimental trials. The second block consisted of two practice trials and nine experimental trials.

\section{Results}

An average of 55.8 probes were presented per experimental trial, resulting in an average of 1,004 probes per observer. The smallest number of probes that occurred on any trial for any observer was 50, and the greatest number was 61 . Only trials on which no more than 9 false alarms were recorded were included in the probe detection analyses. Thus, an average of 0.7 trials, or 39.2 probe events, were excluded per observer. An average of 1.9 false alarms occurred per tracking trial for trials included in the probe detection analyses.

An ANOVA showed a significant main effect of probe location $[F(1.9,36.5)=68.07, p<.001]$. Bonferroni post hoc tests were used to test for differences between the locations. The miss rates were lower for the probes occurring on the center $(M=.21, S D=.10)$ than for those on End $1(M=.35, S D=.11)(p<.001)$. The miss rates were also lower for the probes occurring on the center than for those on End $2(M=.40, S D=.11)(p<.001)$. The miss rates for the probes at End 1 did not differ from those at End $2(p>.05)$.

The main effect of high-probability location was not significant $[F(1,19)=0.03, p>.05]$. The miss rates in 
the End 1 high-probability condition $(M=.32, S D=.11)$ did not differ from those in the center high-probability condition $(M=.32, S D=.10)$.

Importantly, as in the other experiments, a significant interaction was found between probe location and highprobability location $[F(1.8,33.3)=5.69, p<.05]$. This interaction can be seen in Figure 6. Bonferroni comparisons indicated that in the center high-probability condition, the center probes had lower miss rates than either End 1 probes $(p<.001)$ or End 2 probes $(p<.001)$, whereas End 1 probes did not differ from the End 2 probes $(p>.05)$. However, in the End 1 high-probability condition, the End 1 probes had lower miss rates than the End 2 probes $(p<.01)$ but higher miss rates than the center probes $(p<.01)$.

\section{Discussion}

The similarity of the results of Experiment 4 to those of Experiments 1 and 2 again indicates attentional enhancement of high-probability locations, as well as the presence of the attentional center bias. This result demonstrates that attention can maintain two different patterns of spatial prioritizations on two objects simultaneously. The finding that two entirely different patterns of attentional prioritization can be maintained concurrently on different moving objects lends further support to the idea that these prioritizations occur within object-based reference frames.

It might be contended that in Experiment 4, the observers maintained a pattern of prioritization only on one of the two objects on each trial, not on both objects. Averaging over multiple trials, this could lead to a pattern suggesting some amount of learning of both patterns. However, if this were the case, the effect of prioritizations should be sub-

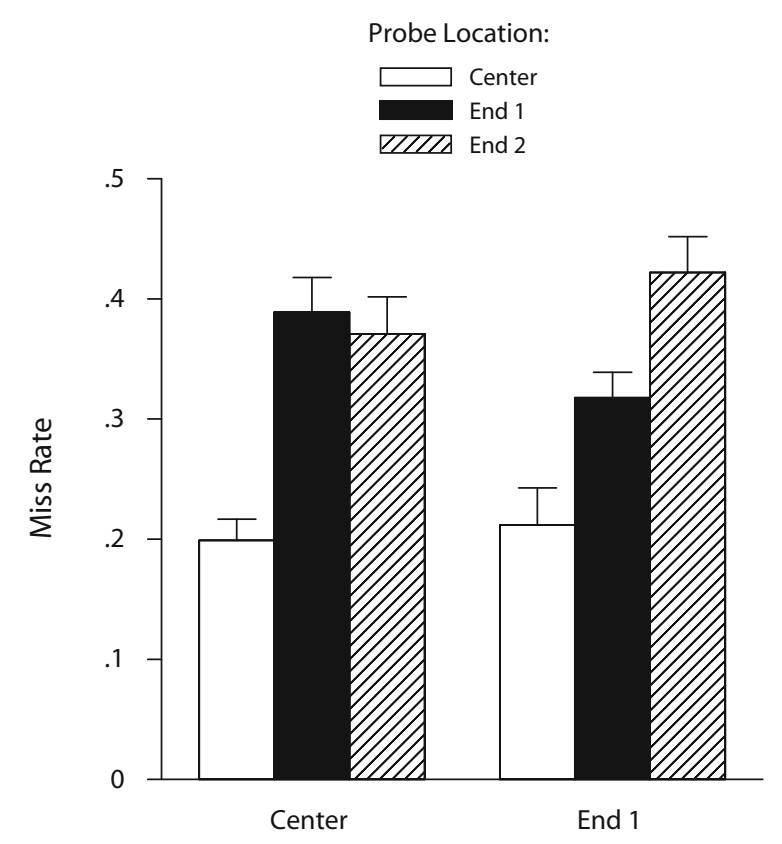

High-Probability Location

Figure 6. Miss rate as a function of high-probability location and probe location in Experiment 4. stantially less in Experiment 4 than that in Experiments 1 and 2, which does not appear to have occurred. Thus, we believe this account to be unlikely, although it cannot be definitively rejected on the basis of the present results.

\section{GENERAL DISCUSSION}

Much research has demonstrated that attention is prioritized to spatial locations on the basis of the probabilities of stimuli appearing at each location (e.g., Geng \& Behrmann, 2005; Hoffmann \& Kunde, 1999; Miller, 1988). The goal of the present study was to examine whether attention can maintain these spatial prioritizations in multiple independently moving reference frames.

Experiment 1 established the principal finding, that attention can maintain spatial prioritizations on moving objects over time. Detection of probes on moving objects was facilitated at locations that had a higher probability of containing probes. Unlike in Feria (2008), in the present stimuli, the high-probability locations were not denoted by any object feature, so the effect cannot be explained by attention to particular object features. Because facilitation occurred at high-probability locations relative to the object, not relative to the display screen, this suggests that the prioritizations occur in an object-based reference frame.

Probe detection was also facilitated at the objects' centers. This supports Alvarez and Scholl's (2005) and Feria's (2008) finding of an attentional bias toward the centers of objects during MOT. Previous research with stationary stimuli has demonstrated an attentional bias toward the center of an array (Zhou et al., 2006) and a tendency for a saccadic eye movement to an object to land near the center of the object (e.g., Vishwanath \& Kowler, 2003, 2004). The attentional bias toward the centers of objects appears to be a pervasive and robust phenomenon.

Experiment 2 demonstrated that if the observer is not given information about the location probabilities on the moving objects, the probability distribution can be learned. Previous studies in which stationary displays were used have shown that observers can learn probability distributions and prioritize attention accordingly (e.g., Geng \& Behrmann, 2002, 2005; Hoffmann \& Kunde, 1999; Kunde \& Hoffmann, 2005; Miller, 1988). The present study extends this result, showing that observers can learn distributions of location probabilities on rotating and translating objects and prioritize attention according to the probabilities. This finding indicates that the visual system is sensitive to redundancies in the spatial distribution of stimuli, even when the stimuli occur within a rotating and translating reference frame. These stimulus expectancies result in attentional prioritizations relative to the reference frame in which the stimulus redundancies occur. An interesting area for future research will be to examine how the occurrence of a stimulus on an object at a particular orientation and location is associated with the occurrence of a stimulus on the object when it is at a different orientation and location. Perhaps interpolation and extrapolation processes are used to generalize from previously viewed angles and locations to new angles and locations (e.g., Spetch \& Friedman, 2003). 
Experiment 3 examined attentional prioritizations on moving objects that were composed of multiple regions. The results showed that the attentional center bias was smaller for multiple-region objects than for single-region objects. This suggests that the process of representing the location of an object at its center point and biasing attention to that point requires the use of the object representation produced by the perceptual organization principles. A multiple-region object has a less cohesive object representation and thus produces a weaker bias toward its center. This result supports previous research that has shown that attention is affected by uniform connectedness of objects (Lamy \& Egeth, 2002; Matsukura \& Vecera, 2006; Watson \& Kramer, 1999). The present study extends the prior research by demonstrating that uniform connectedness affects attentional processes on moving objects, as well as stationary objects. This is noteworthy because it might have been expected that the grouping principle of common fate (Wertheimer, 1938) would cause a moving multiple-region object to be selected as a unit and, thus, that attentional processes on moving objects would not be affected by uniform connectedness. Apparently, common fate does not completely supersede the lack of uniform connectedness.

Critically, the magnitude of attentional prioritization by probability did not differ between multiple-region objects and single-region objects. Uniform connectedness does not affect attentional prioritization by probability. This result suggests that when spatially prioritizing attention within an object-based reference frame, the top-down demands encourage selection of the object in its entirety, overriding the lack of uniform connectedness. This finding supports previous research showing that top-down demands play a role in whether the unit that attention selects comprises a single region or multiple regions (Watson \& Kramer, 1999).

Experiment 4 showed that when two moving objects are viewed, each having a distinct distribution of spatial probabilities within it, attentional prioritizations are maintained on each object according to its individual distribution. Previous researchers have found that observers could concurrently establish different attentional prioritizations for two different stationary configurations (Hoffmann \& Kunde, 1999; Kunde \& Hoffmann, 2005), and the present study extends this finding to moving objects. The fact that different attentional prioritizations by probability can simultaneously be applied to different moving objects lends further support to the idea that the prioritizations occur within object-based reference frames. An interesting question for future research is whether attentional prioritizations based on spatial probabilities can be maintained on more than two objects simultaneously. Because of the capacity constraints of VSTM (e.g., Luck \& Vogel, 1997), it is expected that attentional prioritizations by probability can be maintained only on a limited number of objects.

Taken together, the results of the four experiments support the hypothesis that attention can maintain spatial prioritizations in multiple independently moving reference frames. This corroborates previous research showing that attentional prioritizations are based on relative position within a configuration, as well as absolute position relative to the display screen (Hoffmann \& Kunde, 1999; Kunde \& Hoffmann, 2005; Miller, 1988). The present results extend those findings by showing that attentional prioritizations within an object can follow the object as it undergoes continuous motion. Thus, the present results provide strong evidence that spatial prioritizations can occur within an object-based reference frame.

The findings of the present experiments fit well with the framework of object-based and space-based attention proposed by Shomstein and Yantis (2004). According to this model, attention can be allocated according to two prioritization schedules, which function by covertly scanning individual locations in a strategic order. The configural mechanism reflects object segmentation of the scene by the principles of perceptual organization, and it assigns higher priority to regions within an attended object than to regions outside of that object. The context-dependent mechanism assigns higher priority to particular locations on the basis of the current behavioral task contingencies, such as probabilities. The present study demonstrates that attentional prioritizations based on probabilities can follow an object as it moves through space. This extends Shomstein and Yantis's idea of the context-dependent mechanism, suggesting that the context-dependent mechanism can allocate attention not only to fixed locations in space that are of high priority, but also to locations on a moving object.

One interesting finding of this study is that multipleregion objects produce less attentional center bias than do single-region objects, but the magnitude of the attentional prioritization by probabilities does not differ for multipleregion objects and single-region objects. This result can be understood within Shomstein and Yantis's (2004) framework if we assume that the center bias is a result of the configural mechanism. Alvarez and Scholl (2005) proposed that the center bias occurs because the visual system needs to represent the location of an extended object at a single point. While an object is viewed over a period of time, the configural mechanism might assign the center of the object as the location to be scanned first and most often. Since the configural mechanism is dependent on object segmentation, less perceptually cohesive multiple-region objects will produce a weaker configural component and, thus, a smaller center bias. On the other hand, prioritization by probabilities is a result of the context-dependent mechanism, which is not dependent on object segmentation and is thus not reduced for multiple-region objects. Future research is needed to test this explanation.

It is worth noting that other authors have asserted that attention does not function as a single focus that rapidly scans items and, instead, have proposed that attention is a parallel process in which locations compete for processing capacity (e.g., Desimone \& Duncan, 1995). According to this view, attention improves the quality of the sensory representation for locations within a previously attended object and for locations with high probability (e.g., Chen $\&$ Cave, 2008). The results of the present study are consistent with either a prioritized scanning process or a sensory enhancement process. 
Although some previous research has suggested that either object-based or space-based attention is used individually, depending on the type of task (e.g., Vecera \& Farah, 1994), other studies have suggested that objectbased and space-based attention can be used concurrently in the same task (e.g., Egly et al., 1994; Shomstein \& Yantis, 2004; Soto \& Blanco, 2004). The present results support the idea that object-based and space-based attention can be used concurrently. Attentional prioritizations based on spatial probabilities can move along with multiple continuously moving objects. These results suggest that space-based prioritizations can occur in an object-based reference frame. Object-based and space-based attention are not independent processes but are highly interactive and linked.

\section{AUTHOR NOTE}

This research was supported by a California State University Research Funds grant, a San Jose State University College of Social Sciences Research Grant, and a San Jose State University Department of Psychology Research Grant. Part of this research was presented at the annual meeting of the Vision Sciences Society, Naples, Florida, May 2009. I thank Shahin Najafi, Tyler Florence, Brenda Serna, Justin Shaw, Rachel Horne, and Deepa Dinakar for assistance with data collection and useful conversations. For computer programming help, I thank Michael Chen and Harpreet Bola. I thank Gilbert Remillard for helpful advice and discussions. I also thank Todd Horowitz for his valuable suggestion, which motivated Experiment 2. Correspondence concerning this article should be addressed to C. S. Feria, Department of Psychology, San Jose State University, One Washington Square, San Jose, CA 95192-0120 (e-mail: cary.feria@sjsu.edu).

\section{REFERENCES}

Alvarez, G. A., \& Scholl, B. J. (2005). How does attention select and track spatially extended objects? New effects of attentional concentration and amplification. Journal of Experimental Psychology: General, 134, 461-476.

BAYLIS, G. C., \& Driver, J. (1993). Visual attention and objects: Evidence for hierarchical coding of location. Journal of Experimental Psychology: Human Perception \& Performance, 19, 451-470.

Chen, Z., \& Cave, K. R. (2008). Object-based attention with endogenous cuing and positional certainty. Perception \& Psychophysics, 70, 1435-1443.

Chua, K.-P., \& Chun, M. M. (2003). Implicit scene learning is viewpoint dependent. Perception \& Psychophysics, 65, 72-80.

Desimone, R., \& Duncan, J. (1995). Neural mechanisms of selective visual attention. Annual Review of Neuroscience, 18, 193-222.

Duncan, J. (1984). Selective attention and the organization of visual information. Journal of Experimental Psychology: General, 113, 501517.

EgLy, R., Driver, J., \& Rafal, R. D. (1994). Shifting visual attention between objects and locations: Evidence from normal and parietal lesion subjects. Journal of Experimental Psychology: General, 123, 161-177.

FERIA, C. S. (2008). The distribution of attention within objects in multiple-object scenes: Prioritization by spatial probabilities and a center bias. Perception \& Psychophysics, 70, 1185-1196.

Geng, J. J., \& Behrmann, M. (2002). Probability cuing of target location facilitates visual search implicitly in normal participants and patients with hemispatial neglect. Psychological Science, 13, 520-525.

Geng, J. J., \& Behrmann, M. (2005). Spatial probability as an attentional cue in visual search. Perception \& Psychophysics, 67, 12521268.

Hoffmann, J., \& Kunde, W. (1999). Location-specific target expectancies in visual search. Journal of Experimental Psychology: Human Perception \& Performance, 25, 1127-1141.

Kahneman, D., \& Treisman, A. (1984). Changing views of attention and automaticity. In R. Parasuraman \& D. R. Davies (Eds.), Varieties of attention (pp. 29-61). New York: Academic Press.

Kahneman, D., Treisman, A., \& Gibbs, B. J. (1992). The reviewing of object files: Object-specific integration of information. Cognitive Psychology, 24, 175-219.

KINCHLA, R. A. (1977). The role of structural redundancy in the perception of visual targets. Perception \& Psychophysics, 22, 19-30.

Kunde, W., \& Hoffmann, J. (2005). Selecting spatial frames of reference for visual target localization. Experimental Psychology, 52, $1-12$.

LAmy, D., \& Egeth, H. (2002). Object-based selection: The role of attentional shifts. Perception \& Psychophysics, 64, 52-66.

LAMY, D., \& TsaL, Y. (2000). Object features, object locations, and object files: Which does selective attention activate and when? Journal of Experimental Psychology: Human Perception \& Performance, 26, 1387-1400.

Lassaline, M. E., \& Logan, G. D. (1993). Memory-based automaticity in the discrimination of visual numerosity. Journal of Experimental Psychology: Learning, Memory, \& Cognition, 19, 561-581.

LUCK, S. J., \& VogeL, E. K. (1997). The capacity of visual working memory for features and conjunctions. Nature, 390, 279-281.

Matsukura, M., \& Vecera, S. P. (2006). The return of object-based attention: Selection of multiple-region objects. Perception \& Psychophysics, 68, 1163-1175.

MiLLER, J. (1988). Components of the location probability effect in visual search tasks. Journal of Experimental Psychology: Human Perception \& Performance, 14, 453-471.

OgaWA, H., TAKeda, Y., \& YAGI, A. (2002). Inhibitory tagging on randomly moving objects. Psychological Science, 13, 125-129.

OLSON, I. R., \& JiAng, Y. (2002). Is visual short-term memory object based? Rejection of the "strong-object" hypothesis. Perception \& Psychophysics, 64, 1055-1067.

PaLmer, S. E. (1992). Common region: A new principle of perceptual grouping. Cognitive Psychology, 24, 436-447.

PALMER, S. [E.], \& Rock, I. (1994). Rethinking perceptual organization: The role of uniform connectedness. Psychonomic Bulletin \& Review, 1, 29-55.

Posner, M. I., SNyder, C. R., \& Davidson, B. J. (1980). Attention and the detection of signals. Journal of Experimental Psychology: General, 109, 160-174.

Prinzmetal, W., McCool, C., \& Park, S. (2005). Attention: Reaction time and accuracy reveal different mechanisms. Journal of Experimental Psychology: General, 134, 73-92.

Pylyshyn, Z. W., \& Storm, R. W. (1988). Tracking multiple independent targets: Evidence for a parallel tracking mechanism. Spatial Vision, 3, 179-197.

Scholl, B. J. (2001). Objects and attention: The state of the art. Cognition, 80, 1-46.

Shaw, M. L., \& Shaw, P. (1977). Optimal allocation of cognitive resources to spatial locations. Journal of Experimental Psychology: Human Perception \& Performance, 3, 201-211.

Shomstein, S., \& Yantis, S. (2004). Configural and contextual prioritization in object-based attention. Psychonomic Bulletin \& Review, 11, 247-253.

Soto, D., \& Blanco, M. J. (2004). Spatial attention and object-based attention: A comparison within a single task. Vision Research, $\mathbf{4 4}$ 69-81.

Spetch, M. L., \& Friedman, A. (2003). Recognizing rotated views of objects: Interpolation versus generalization by humans and pigeons. Psychonomic Bulletin \& Review, 10, 135-140.

Tipper, S. P., Brehaut, J. C., \& Driver, J. (1990). Selection of moving and static objects for the control of spatially directed action. Journal of Experimental Psychology: Human Perception \& Performance, 16, 492-504.

Treisman, A. M., \& Gelade, G. (1980). A feature-integration theory of attention. Cognitive Psychology, 12, 97-136.

VECERA, S. P., \& FARAH, M. J. (1994). Does visual attention select objects or locations? Journal of Experimental Psychology: General, 123, 146-160.

Vishwanath, D., \& Kowler, E. (2003). Localization of shapes: Eye movements and perception compared. Vision Research, 43, 16371653. 
Vishwanath, D., \& Kowler, E. (2004). Saccadic localization in the presence of cues to three-dimensional shape. Journal of Vision, $\mathbf{4}$, $445-458$.

Watson, S. E., \& Kramer, A. F. (1999). Object-based visual selective attention and perceptual organization. Perception \& Psychophysics, 61, 31-49.

WertheImer, M. (1938). Laws of organization in perceptual forms. In W. D. Ellis (Ed.), A sourcebook of Gestalt psychology (pp. 71-88). London: Routledge. (Original work published 1923)

Wolfe, J. M., Cave, K. R., \& Franzel, S. L. (1989). Guided search: An alternative to the feature integration model for visual search. Journal of Experimental Psychology: Human Perception \& Performance, 15, 419-433.
Zhou, X., ChU, H., LI, X., \& ZHAN, Y. (2006). Center of mass attracts attention. NeuroReport, 17, 85-88.

\section{NOTE}

1. The reaction time results resembled the miss rate data, indicating that there was no speed-accuracy trade-off.

(Manuscript received August 13, 2009;

revision accepted for publication January 9, 2010.) 